Document downloaded from:

http://hdl.handle.net/10251/120931

This paper must be cited as:

Alfonso Laguna, CD.; Caballer Fernández, M.; Calatrava Arroyo, A.; Moltó, G.; Blanquer Espert, I. (2018). Multi-elastic Datacenters: Auto-scaled Virtual Clusters on Energy-Aware Physical Infrastructures. Journal of Grid Computing. 17(1):191-204.

https://doi.org/10.1007/s10723-018-9449-z

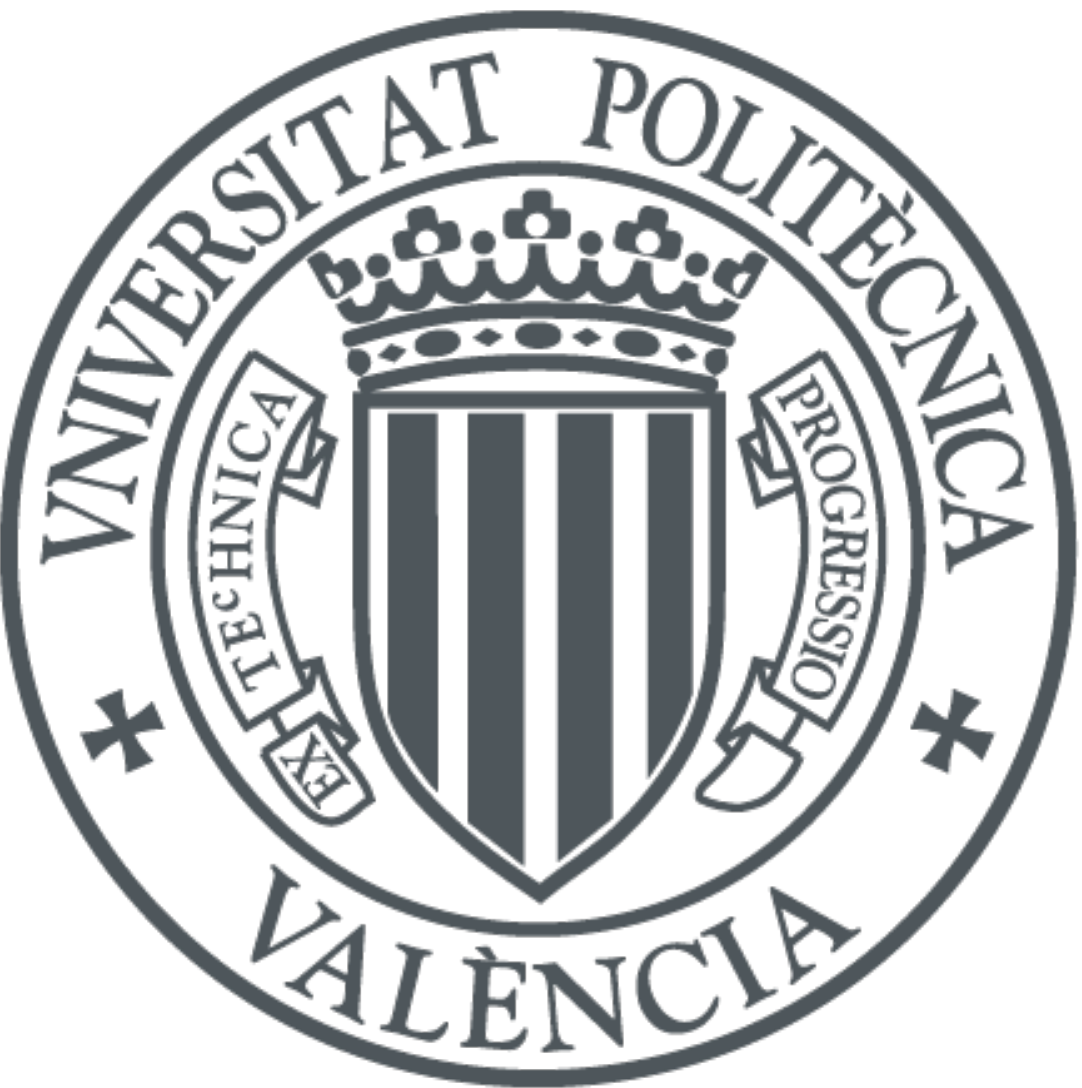

The final publication is available at

http://doi.org/10.1007/s10723-018-9449-z

Copyright Springer-Verlag

Additional Information 


\title{
Multi-Elastic Datacenters: Auto-Scaled Virtual Clusters on Energy-Aware Physical Infrastructures
}

\author{
Carlos de Alfonso ${ }^{\mathrm{a}, *}$, Miguel Caballer ${ }^{\mathrm{a}}$, Amanda Calatrava ${ }^{\mathrm{a}}$, Germán Moltóa ${ }^{\mathrm{a}}$, Ignacio \\ Blanquer ${ }^{\mathrm{a}}$ \\ ${ }^{a}$ Instituto de Instrumentación para Imagen Molecular (I3M) \\ Centro mixto CSIC - Universitat Politècnica de València \\ Camino de Vera s/n, 46022, Valencia
}

\begin{abstract}
Computer clusters are widely used platforms to execute different computational workloads. Indeed, the advent of virtualization and Cloud computing has paved the way to deploy virtual elastic clusters on top of Cloud infrastructures, which are typically backed by physical computing clusters. In turn, the advances in Green computing have fostered the ability to dynamically power on the nodes of physical clusters as required. Therefore, this paper introduces an open-source framework to deploy elastic virtual clusters running on elastic physical clusters where the computing capabilities of the virtual clusters are dynamically changed to satisfy both the user application's computing requirements and to minimise the amount of energy consumed by the underlying physical cluster that supports an on-premises Cloud. For that, we integrate: i) an elasticity manager both at the infrastructure level (power management) and at the virtual infrastructure level (horizontal elasticity); ii) an automatic Virtual Machine (VM) consolidation agent that reduces the amount of powered on physical nodes using live migration and iii) a vertical elasticity manager to dynamically and transparently change the memory allocated to $\mathrm{VMs}$, thus fostering enhanced consolidation. A case study based on real datasets executed over a production infrastructure is used to validate the proposed solution. The results show that a multi-elastic virtualized datacenter provides users with the ability to deploy customized scalable computing clusters while reducing its energy footprint.
\end{abstract}

Keywords: Cloud Computing, Green computing, Elasticity

\section{Introduction}

Computer clusters are a very common computing facility used both for scientific institutions and enterprises. A cluster consists of a set of computing nodes connected using at least one high-speed low-latency network and it is usually managed by a Local Resource Management System (LRMS) used to manage the whole lifecycle of the jobs.

\footnotetext{
*Corresponding author: Tel. +34963877356

Email address: caralla@upv.es (Carlos de Alfonso)
} 
These jobs typically represent different workloads such as High Throughput Computing (HTC) or High Performance Computing (HPC).

However, physical clusters face several drawbacks. Firstly, they require a significant capital investment together with the costs required for housing and periodic hardware maintenance. Secondly, maintaining a physical cluster up and running on a $24 / 7$ basis in order to deliver full level of service, when it is required, is very expensive, mainly due to the cost of energy [15. Indeed, the energy is consumed both by the cluster itself and the cooling system required to maintain the environmental conditions. These physical clusters are typically over-dimensioned to cope with increased workloads and, specially, peaks of demand. However, these peaks are rarely reached while underutilization is a very often scenario. Indeed, as Williams et al. [40] described, in well-provisioned datacenters, overload is unpredictable, relatively rare, uncorrelated, and transient.

Therefore, one of the challenges for computing clusters is to reduce their energy consumption. The energy saving techniques applied for clusters are basically, Static Power Management (SPM) techniques, which consist of using more efficient components, and Dynamic Power Management (DPM) techniques, that consist of adapting the infrastructure to the actual workload 37. One common DPM approach for computing clusters is to power off those physical nodes that are idle and power them back on again as they are needed. Such energy saving technique has been proved to provide substantial cost reduction in cluster infrastructures in our previous publication [16].

On the other hand, user applications typically have special requirements (libraries, compilers, Operating System (OS) versions, etc.) what leads to potential software conflicts on multi-tenant scenarios where multiple users share the same cluster. Also, virtualization and Cloud Computing have changed the way of managing a datacenter. Many datacenters are creating their on-premises Clouds to manage their servers using a Cloud Management Platform (CMP) such as OpenNebula, OpenStack, VMWare vCenter, etc. Then, the sysadmins create the Virtual Machines (VMs) needed by the users instead of granting access to physical machines. Indeed, Virtual Cluster (VC) use VMs as the computing nodes, which can support the same functionality as their physical counterparts. This way, virtual clusters can be specifically tailored to the hardware, software and configuration requirements of applications to be run on them. The ability to provision customized virtual clusters is beneficial both for the user, who can access computing resources on-demand and for the sysadmin, since these clusters are decoupled from the underlying execution infrastructure and no adaptation of applications to the computing environment is required.

Indeed, users are provided customized virtual clusters running an specific version of an OS and a set of libraries, managed by the preferred LRMS of the user. Providing VCs with a precise hardware and software configuration that matches the requirements of an application better guarantees its successful execution. VCs are provisioned on-demand and terminated when no longer required so that other virtual clusters can be deployed, thus providing the means of multiplexing access to the underlying physical computing resources. Virtual clusters can also benefit from the elasticity of Cloud infrastructure by terminating the idle VMs and provisioning new ones when they are needed. These Elastic Virtual Clusters (EVC) behave as physical clusters where the DPM technique is applied to the cluster to power on or off the nodes. In the case of the EVC, the working nodes are VMs that are deployed or terminated depending on the workload.

However, such dynamism in the creation and destruction of VMs in an on-premises 
Cloud typically leads to a fragmented distribution of the VMs in the physical servers. In this situation, the request to deploy a VM can be denied because no single host has enough physical resources, even though the aggregation of physical resources from different nodes would allow the VM to be deployed. Increasing the consolidation ratio, where VMs are hosted in a fewer number of hosts, would allow the deployment of the VM. Moreover, the physical Cloud platform can also benefit from the aforementioned DPM technique to power off idle servers in order to reduce the overall energy consumption of the on-premises Cloud.

This paper describes the work towards a multi-elastic datacenter in which the users are delivered EVCs that run on top of on-premises Cloud infrastructures supported by elastic energy-aware physical clusters. The physical nodes that are not hosting any VMs are automatically powered on or off, as needed, to introduce elasticity at a physical level. The VMs of the EVC can be vertically scaled, in terms of the allocated memory, according to the dynamic memory consumption patterns of the application (or applications) being executed. These VMs are automatically live-migrated between hosts to increase server consolidation and use a reduced number of physical hosts. Therefore, this creates a multi-elastic datacenter where automated horizontal elasticity is applied for physical and virtual computing resources together with automated vertical elasticity for the EVCs. A set of open-source developments have been released in order to support this vision and deployed in production within our research group.

After the introduction, the paper is organized as follows. First, section 2 discusses the related work in the different areas covered by this paper. Next, section 3 describes the proposed approach together with the building blocks required to build a multi-elastic datacenter. Later, section 4 describes a case study in order to assess the advantages of the proposed approach. Finally, section 5 summarises the paper and points to future work.

\section{Related work}

Apart from the CMP, that manages the lifecycle of VMs running on the aforementioned physical infrastructure, several key components are needed for the multi-elastic datacenter: i) a tool to deploy customized EVCs through VMs managed by the aforementioned CMP and ii) an automated power management system for the physical infrastructure; iii) mechanisms to consolidate the VMs in the CMP in order to increase the VM-per-host ratio; iv) a system to automatically change the memory allocation of the VMs to the dynamic requirements of the applications being executed on them. The following subsections describe the main related works in these areas.

\subsection{Elastic Virtual Clusters}

There are different examples of tools to deploy virtual clusters in the literature, such as [10, 18, or 39. These works mainly deal with the provision of the VMs and configuration of the cluster topology (e.g. connectivity, shared filesystem, ssh-ability, etc.). Some of them include configuration capabilities (e.g. installing applications, creating users, etc.). However, their approach lacks elasticity. In this sense, once a cluster has been delivered to the user, all the VMs will continue running even if they are idle. Such static behaviour may prevent from creating new clusters because of the lack of resources. 
Focusing on elastic clusters, there are several works that address the problem. As an example, [17] and [26] evaluate the possibility of using Amazon EC2 to extend a physical cluster, depending on the workload. In [31, the authors explore the dynamic provision of working nodes in the cloud, depending on the size of the jobs in the queues, introducing several policies to limit the amount of working nodes to be powered on. The main limitation of these works is that they seem to be ad-hoc private implementations that have not been released as open-source components.

Focusing on ready-to-use open-source tools, the standard distribution of Hadoop [5] includes an easy-to-use mechanism to create a virtual cluster in Amazon Web Services (AWS). The main problem is that is exclusively designed for Hadoop and, in addition, the number of nodes for the cluster is not dynamically managed (although it is possible to manually add or destroy working nodes ). StarCluster [29] is an open source cluster-computing toolkit built exclusively for AWS. It uses pre-built Virtual Machine Images (VMI) with specific software installed. It is based on the Open Grid Scheduler LRMS (formerly known as SGE) and includes common libraries such as OpenMPI, OpenBLAS, Lapack, etc. It also features a module called Elastic Load Balancer that supports shrinking or expanding the cluster based on the statistics of the queues of the LRMS. However, the cluster is not self-managed since an external system (typically the user's computer running the StarCluster application) has to monitor the cluster to decide whether elasticity should be performed.

In this way, 3 is a development to create entire virtual clusters running a batch system such as HTCondor that grow and shrink automatically based on the usage, although this requires external continuous monitoring of the cluster. The caveat in this work is that it can only run their Virtual Machine Images, and requires a Cloud platform compliant with the Amazon EC2 interface.

As opposed to previous works, we propose a system that builds entirely on publicly available open-source components in order to automatically manage elasticity both at the physical and the virtual levels, featuring vertical elasticity for virtual clusters. These features, combined with dynamic power management of physical clusters and automated consolidation of VMs via live migration provides the foundation of a multi-elastic datacenter. As far the authors are aware there is no such approach currently available in the literature that addresses simultaneous multi-level elasticity of virtual infrastructures on physical infrastructures.

\subsection{Automated Power Management}

The main CMPs do not offer automated power management out of the box, specially in the open-source versions of the products. For example, OpenQRM ${ }^{1}$ introduces power saving features exclusively for the enterprise version, which is distributed under a commercial license. In the commercial cloud platforms, there are several solutions that offer automated power management features. This is the case of VMWare vSphere 5.5 which is capable of powering on and off physical hosts. However, it is restricted to the VMWare's hypervisor in addition to being very costly (beginning at USD 2,875.00 for the version able to manage the power of the nodes). Huawei's FusionSpher $\AA^{3}$ also offers

\footnotetext{
${ }^{1}$ http://www.openqrm-enterprise.com

${ }^{2}$ http://www.vmware.com/products/vsphere

${ }^{3} \mathrm{http}: / /$ e.huawei.com/en/products/cloud-computing-dc/cloud-computing/fusionsphere/fusionsphere 
automated power management. It builds up on OpenStack, but its solution is also distributed under a commercial license. However, commercial solutions are out of the scope of this paper since once purchased a commercial CMP, the user is typically restricted to the solutions offered by the specific vendor.

\subsection{Facilitating Power Management}

There are works that try to reduce the number of physical servers needed to host the VMs deployed on a Cloud, specially at the scheduler level. In fact, most of the schedulers shipped in the default distributions of the CMPs include features for reducing the number of used servers. However, during the lifecycle of the platform (i.e. sequences of creation and destruction of VMs) the distribution of the VMs may prevent from achieving idle servers even when the running VMs could be hosted in a fewer number of servers.

There are several works that try to profit from live-migration features to consolidate the VMs in a platform into a few number of physical hosts. Some common approaches consist of applying reinforcement learning [33 11] 19, fuzzy logic [28] or nature-based solutions such as the works in 20] (which is inspired on the movements of the ants in a colony), 32] and [27] (that are inspired on the behaviour of the swarms during migratory flights), or [21] (which is based on the movements of a bee colony). Other approach consist of modelling the problem as a multidimensional bin packing $(\mathrm{mBP})$ problem where the physical nodes are modelled as multi-dimensional containers, and each dimension corresponds to a resource (typically CPU, memory or hard disk). In this field there are several proposal of works such as 2] that statically reduces the number of physical hosts, but is not intended to be used in a continuously working platform. The work by Verma et al. 38 tries to combine VM placement with Dynamic Voltage and Frequency Scaling (DVFS). Finally, it is noticeable the work by Beloglazov et al. [4] that solves the bin packing problem and includes a scheduler to take into account energy saving criteria to re-place the VMs, or the works [22] and 34] that also try to reduce the number of used physical hosts.

\subsection{Adapting the VMs to the actual workload}

The users tend to overestimate the amount of memory required by their applications resulting in unused memory that could be dedicated to additional VMs running on the same physical machine [36]. Moreover, CMPs typically offer different instance types, also known as flavours in the case of OpenStack, out of which the VMs are instantiated. These instance types define the amount of memory, cores and storage that will be allocated to the VM. The users select the instance type according to its constraints (e.g. the number of cores) even if they do not need the corresponding amount of memory. Apart from the waste of resources, over-dimensioning a VM also hinders VM consolidation into a reduced number of servers.

Some works have tried to adjust the resources of the VMs to the actual workload. As an example, the work shown in [12] tries to adapt the allocation of the CPU in the VMs running on the Xen hypervisor, but it does not consider changing the memory. There are also other works such as [35] and 23] that try to adapt the virtual memory to the actual needs of the applications running in the VMs using various methods. However, they consider it only at the host level instead of the whole physical infrastructure managed by the CMP. Therefore, the CMP is not able to oversubscribe the hosts considering the 


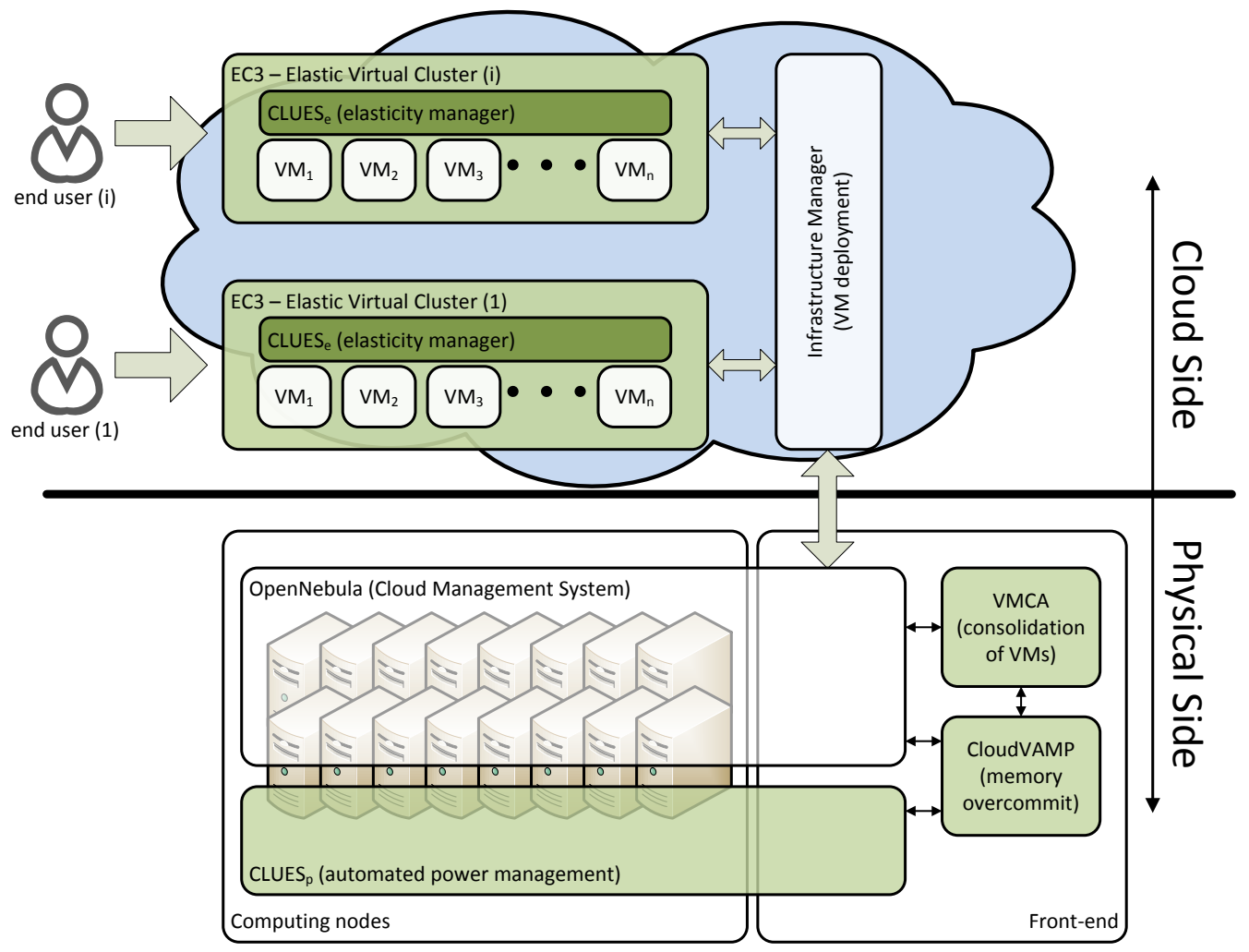

Figure 1: The multi-elastic datacenter building blocks.

memory that is actually being used rather than the memory that is currently allocated. There are also works such as 25] that tackle the problem at the CMP level, but their approach does not provide countermeasures in case the host memory is overcommitted and a VM claims back the memory that it had originally requested.

\section{The Multi-Elastic Datacenter}

The building blocks of the multi-elastic datacenter are summarized in Figure 1. Two layers are clearly distinguished: the Cloud Infrastructure (Physical Side) and the Cloud Services \& Application (Cloud Side). On the one hand, the Cloud infrastructure is managed by the system administrators who are responsible for installing and managing both the physical servers and the CMP. On the other hand, the Cloud Services \& Application layer provides the user with the EVCs on which applications/jobs are executed.

\subsection{The Cloud Infrastructure Layer}

The Cloud infrastructure comprises a set of physical servers arranged as an onpremises Cloud platform and managed by a CMP such as OpenNebula or OpenStack. 
The following components have to be installed by the system administrator to introduce the ability of dynamic power management, i.e., elasticity at the physical level:

- CLUster Energy Saving (CLUES) 14 which is an automated power manager for computer clusters. It also supports plugins to integrate with CMPs such as OpenNebula, in order to power on and off the physical nodes depending on the requirements. For physical computer clusters, CLUES monitors the LRMS to decide when additional worker nodes have to be powered on according to different reactive policies. For virtual clusters, the semantics of powering on and off the nodes have been changed into deployment and termination of VMs. This way, CLUES can dynamically provision and relinquish VMs from a Cloud provider. For CMPs, CLUES intercepts the VM deployment requests to decide if physical nodes should be powered on. Using the same framework for elasticity at those three levels enable to reuse policies and maintain a consistent behaviour across the different layers.

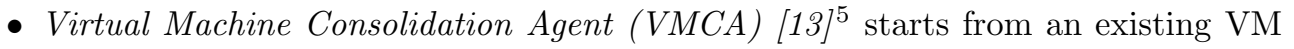
distribution within an on-premises Cloud and produces a migration plan in order to achieve a set of idle nodes for CLUES to power them off. Live migrations are performed with the support provided by the KVM hypervisor and it has been integrated with the OpenNebula CMP.

- Cloud Virtual Machine Automatic Memory Procurement (CloudVAMP 6 [30] is an automatic system that enables and manages memory over-subscription in an onpremises OpenNebula Cloud platforms. Using active monitoring of the VMs and considering the actual memory used by the VM, regardless of the initially allocated memory, it dynamically resizes the memory of the running VMs without downtime by leveraging memory ballooning techniques provided by the KVM hypervisor. Fully integrated with the CMP, it lets OpenNebula deploy additional VMs per server thus increasing the VM-per-host ratio and, as a side product, letting VMCA to obtain more idle servers. Live migration is employed to prevent memory overload of the physical hosts in order to restore the level of service.

The interface between the Cloud infrastructure and the Cloud services \& application is the CMP. Apart from the common administrative tasks in the platform (e.g. creating OS disks, managing users and permissions, creating subnetworks, etc.), the interaction from the Cloud consists of creating or destroying VMs. The events that may happen in the physical side as a result from the creation or destruction of VMs are described below:

- When new VMs are created and there are not enough resources (typically in terms of memory or number of virtual CPUs) for them, $C L U E S_{p}$ will power on one or more physical nodes to provide the required resources to run the new VMs. The VMs deployment request are held by the CMP until the physical servers are powered on. When the physical nodes are finally available, the VMs are deployed, and they will be started by the CMP on the appropriate host(s).

\footnotetext{
${ }^{4}$ CLUES - http://www.grycap.upv.es/clues

${ }^{5}$ VMCA - http://www.grycap.upv.es/vmca/

${ }^{6}$ CloudVAMP - http://www.grycap.upv.es/cloudvamp 
- When physical servers become idle, either because the VMs terminate or VMCA has migrated them to other physical nodes, $C L U E S_{p}$ will power them off to save energy.

- If a VM $j$ is not using the memory requested during its creation, CloudVAMP will dynamically reduce its allocated memory without any downtime for the VM, according to the vertical elasticity rules described in [30].

- If a VM $j$ whose memory was reduced requires it, e.g. the application running on the VM starts demanding more memory, CloudVAMP will check if the server in which it is hosted has enough free physical memory. If not, CloudVAMP will request $C L U E S_{p}$ to power on a new physical server, and once the server is available, the VM will be live migrated to it. Once the VM is in a host that has enough free physical memory, CloudVAMP will increase the memory of the VM, according to the vertical elasticity rules.

- If some VMs are in a physical host $h_{t}$ but they can be hosted among other servers without compromising the quality of service, VMCA will prepare and execute a migration plan to get the host $h_{t}$ free. Once the VMs are migrated, $C L U E S_{p}$ will power off the physical node, since the host $h_{t}$ became idle.

\subsection{The Cloud Services \& Applications Layer}

Users deploy their EVCs by means of EC3 (Elastic Cloud Computing Cluster) ${ }^{7}[9$. This is a tool to deploy self-managed cost-efficient elastic virtual clusters on top of Cloud platforms. It supports different LRMS such as SLURM, Torque, Mesos and SGE. EC3 relies on the Infrastructure Manager ${ }^{8}$ [7] to provision the VMs on multiple back-ends, including, but not limited to, public Clouds such as Amazon Web Services and onpremises Clouds such as OpenNebula and OpenStack. EC3 deploys a front-end node with CLUES specifically configured to be able to deploy and terminate VMs, instead of dealing with physical hosts. This is called $C L U E S_{e}$ in Figure 1. This way, when additional worker nodes are required, new VMs are automatically deployed up to a user-specified maximum on the Cloud platform. It also includes support for hybrid deployments across multiple Clouds (either on-premises and/or public), migration capabilities and automatic checkpointing together with cost-efficient mechanisms such as the usage of spot instances, a potentially cost-reducing instance type available in Amazon EC2.

Once the user has deployed an EVC $i$, some events will happen on this layer:

- When the user submits new jobs to the cluster, $C L U E S_{e}^{i}$ will check if there are enough free working nodes to execute the job. If this is not the case and the maximum cluster size has not been reached, it will ask the IM to deploy additional VMs to be integrated as new working nodes in the cluster in the LRMS. Notice that this procedure is transparent to the user, who only notices a delay since the time the job is submitted to the LRMS and the time the job starts executing.

- When the working nodes become idle for a while, $C L U E S_{e}^{i}$ will terminate the corresponding VMs in order to free the used computing resources.

\footnotetext{
${ }^{7}$ EC3 - http://www.grycap.upv.es/ec3

${ }^{8} \mathrm{IM}-$ http://www.grycap.upv.es/im
} 
CLUES support multiple customizable elasticity rules, described deeply in [14. As an example, CLUES can be configured to power on single nodes or groups of nodes based on a sensor system, and power them off when they are idle a configuring period of time.

\subsection{Complex actions in the Multi-Elastic Datacenter}

During the lifecycle of the multi-elastic datacenter, some complex actions may be triggered as a result of the interaction of the user with the clusters in the Cloud. These complex actions are the result of the interaction of the different building blocks of the multi-elastic data center. Examples of such actions are summarized below:

- A user submits a job to cluster $i$. $C L U E S_{e}^{i}$ detects that there are not free working nodes and requests a VM to the $I M$. The $I M$ deploys a new VM through the CMP. $C L U E S_{p}$ detects that there are not free physical servers and powers on a new one. When the physical host is on, the VM is started and the $I M$ can integrate the VM in the cluster $i$. The job can finally be started. Again, this process is completely transparent for the user.

- A VM which is part of the cluster $i$ is not using the memory requested. CloudVAMP reduces the size of the memory of the VM. The VM is alone in one of the physical servers, but VMCA detects that it can fit into other server. Therefore, VMCA live-migrates the VM, and now the physical host is idle. If the server is still idle after for a certain amount of time, $C L U E S_{p}$ powers off the physical server.

- A VM $\left(V M_{j}^{i}\right)$ whose memory was downsized, in the cluster $i$, starts using the memory again. CloudVAMP detects that there is not enough allocated physical memory in the server in which the $\mathrm{VM}$ is hosted and requests $C L U E S_{p}$ to power on a new physical server. When the physical server is available, CloudVAMP livemigrates $V M_{j}^{i}$ to the new server and resizes the granted memory.

All the aforementioned components are distributed as open-source and made available in GitHulf9 Additionally, some of the components have been adopted in large-scale research infrastructures. In particular, the IM has been integrated in the VMOps Dashboard of EGI (European Grid Infrastructure), see [ 6 for details, while EC3 has been integrated in the EGI Access service to provide Virtual Elastic Clusters as a Service for the Long Tail of Science (LToS), see [8] for details.

\section{Case Study}

This section describes a case study in order to validate the proposed solution to produce multi-elastic datacenters on realistic settings. For this, we adopted real workloads obtained form the Grid Workloads Archive 24] and considered an scenario where two virtual elastic clusters were deployed by means of EC3 on the same Cloud infrastructure configured with the aforementioned tools. Both clusters executed the same job submission pattern. The second virtual cluster $(\mathrm{C} 2)$ was created three hours after the first one (C1). The maximum size of each virtual cluster was fixed to 12 nodes, considering the

${ }^{9}$ GitHub Organization: https://github.com/grycap 


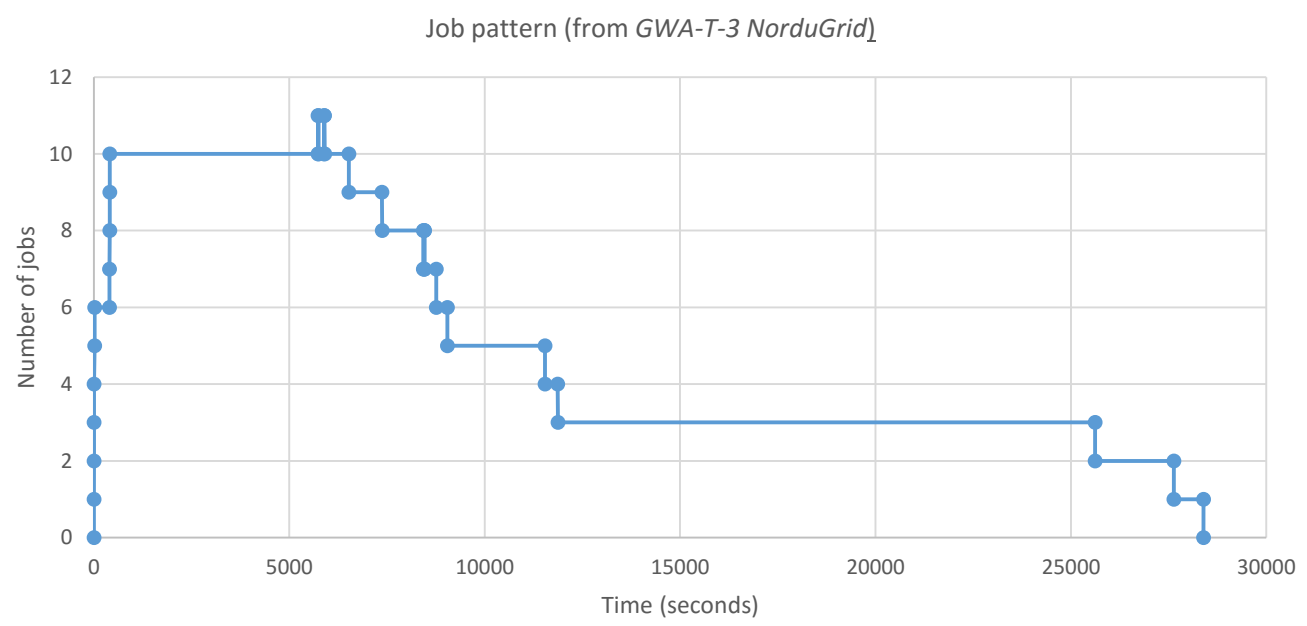

Figure 2: Workload of the case study, extracted from the GWA-T-3 NorduGrid dataset. The blue line represents the evolution of the workload in terms of number of jobs.

workload used. The following configuration was specified for $C L U E S_{e}$, i.e., the elasticity manager of the virtual clusters: i) no limit to the number of nodes concurrently provisioned and ii) idle nodes where powered off after 600 seconds.

The underlying physical infrastructure employed is used in production and during the execution of the case study, $C L U E S_{p}$, i.e., the energy-aware elasticity manager, did not have to power on physical nodes on which the VMs of the virtual clusters would be running. Notice that the only effect in the case study would be an increased time since a VM is deployed until the VM is up and running to include the time required to power on the physical node.

Concerning memory ballooning, CloudVAMP was configured to keep a minimum memory size per VM of $384 \mathrm{MB}$, required for the Operating System to properly function and allowing each node to maintain a $30 \%$ of free memory, which corresponds to a Memory Overprovisioning Percentage (MOP) slightly increased compared to the $20 \%$ value used in our earlier work in vertical elasticity [30. Indeed, for this case study, the amount of cluster reconfigurations caused by adding additional nodes to the clusters introduced additional memory usage peaks which were better accommodated by these increased free memory safety margin.

The real workload, used in both cluster executions, is a fragment extracted from the GWA-T-3 NorduGrid dataset offered by the Grid Workloads Archive (from line 4 to line 16 of the .gwf file), and represented in Figure 2 in terms of number of jobs. For the sake of reproducibility of results, the duration of each job has been reduced up to a $200 \%$. The jobs executed in that dataset are 13 sequential tasks [1, with an average duration of 186 min. In our case, we used a synthetic memory-consuming application ${ }^{10}$ that is able to reproduce a pattern of memory usage that consists of three periods, (i) increasing from 0

${ }^{10}$ https://github.com/grycap/synthalloc 
$\mathrm{MB}$ to $500 \mathrm{MB}$, (ii) maintaining the consumption in those $500 \mathrm{MB}$ of memory, and (iii) reducing the usage of memory from $500 \mathrm{Mb}$ to $0 \mathrm{Mb}$. This behaviour can be appreciated in Figure 3, where the granted memory for a node of the cluster $\mathrm{C} 1$ is represented. The rationale behind the pattern is to use a dynamic memory consumption pattern in order to trigger the activation of CloudVAMP for the adjustment of the allocated memory to the VMs.

The underlying physical infrastructure used is composed by an heterogeneous bladebased system that has four kind of nodes: 2 x (2 quad-core L5430@2.6 Ghz, 16 GB), 2 x (2 quad-core multithreaded E5520@2.26 GHz, $16 \mathrm{~GB}), 6 \mathrm{x}$ (2 quad-core multithreaded E5620@2.4 GHz, $16 \mathrm{~GB}$ ) and $3 \mathrm{x}$ (4 quad-core multithreaded E7520@1.86 GHz, 64 GB), with a total amount of 128 cores and 352 GB of RAM. The blade system is backed by a 16 TB SAN connected via a private gigabit ethernet network. This system is managed by OpenNebula 5.2.1, using KVM as the underlying hypervisor.

For the deployment of the virtual cluster nodes we have relied on pre-configured VMIs, since these introduces a $70 \%$ savings of time in the contextualization phase for each virtual node 9 . Therefore, the VMI selected is based on Ubuntu 14.04 with SLURM 14.11 and NFS pre-installed. Each VM, that corresponds with a node of the cluster, has been deployed with one CPU and requesting $1024 \mathrm{Mb}$ of RAM memory.

Finally, the effects of VMCA were not analysed in this case study since the focus is set on the multi-level elasticity achieved by the integration of a vertical elasticity memory oversubscription tool (CloudVAMP) with a horizontal elasticity manager (CLUES) for the execution of applications with dynamic memory-consuming patterns on virtual elastic clusters. A detailed analysis of VMCA is published in [13].

\subsection{Results}

In this subsection, we first analyse the results obtained from the execution, and then discuss the main contributions of the proposed solution. Figures 3 and 4 cover the main results of the case study.

Figure 3 shows the evolution of the granted memory for a VM of cluster C1. The orange line represents the three different memory consuming phases of the application, as described above, during its execution. Every job has a different duration execution time. The grey line of the graph represents the granted memory to the VM while the blue line represents the initially requested memory of the VM (1024 MB). Indeed, the memory requested when deploying the VM represents an upper bound to the memory allocated at any given time to the VM.

Notice in the figure that a newly deployed VM receives all the requested memory (1024 MB), but as soon as CloudVAMP detects that the VM has free memory beyond the thresholds set by the $30 \%$ MOP, it steals the unused memory in order to make room in the physical node for other VMs to be deployed on that node by the OpenNebula scheduler. The amount of memory borrowed never leaves the VM with less than $384 \mathrm{MB}$ of RAM and less that $30 \%$ of free memory.

The spiky peaks in the granted memory to the VM (gray line) appear to be related to memory consumption by other applications running inside the VM, specially concerning the horizontal elasticity of the virtual cluster, i.e. when the whole cluster is reconfigured by Ansible when a new virtual node is deployed or a virtual node is terminated because no jobs are available for execution. 


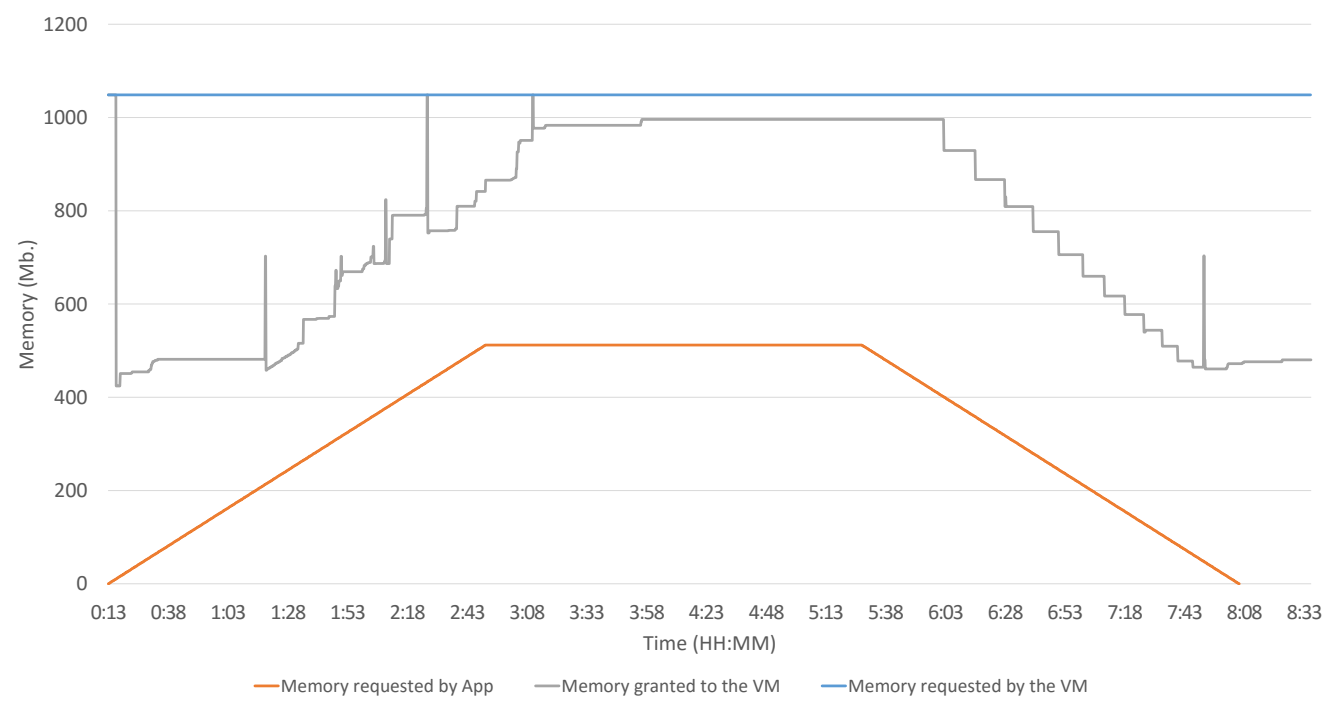

Figure 3: Granted memory of a working node from cluster C1 in contrast with the job requested memory. The orange line represents the memory requested by the job. The grey line represents the memory granted to the VM while the blue line depicts the initial memory requested by the VM.

Finally, the figure shows the grace period (600 s.) that CLUES allocated to the idle virtual node, before terminating it, just in case further jobs were submitted to be deployed in that cluster. This strategy enables to gently accommodate an incoming job without requiring an additional deployment of a virtual node, at the expense of an increased energy consumption in the underlying physical infrastructure. Notice that this grace period can be configured by the user.

Figure 4 describes the evolution of the total requested memory and the memory granted to the different virtual nodes of the two clusters (Figure 4(a) and the evolution of the size of the clusters in terms of number of nodes, i.e. VMs (Figure 4(b)).

Figure 4(a) differentiates the results for cluster $\mathrm{C} 1$, where each blue line represents the granted memory to each node of the cluster, and for cluster $\mathrm{C} 2$, where red lines are used. The light grey area in the background represents the total memory assigned for both clusters during the execution, managed by CloudVAMP. This tool was able to dynamically and transparently change the memory allocated to VMs depending on the current workload. Its effect can be noticed when comparing the grey area with the blue and red lines, where the first represents the total memory initially requested by each VM that compose the clusters (which was $1024 \mathrm{Mb}$ per node) and the second the real assigned memory to each VM

Specifically, CloudVAMP introduced a $29.13 \%$ memory saving, thus allowing increased server consolidation ratio and, thus, better usage of resources.

Figure 4(b) shows the elasticity evolution of both clusters, C1 and C2, in terms of number of nodes. The size of both clusters was dynamically adapted to their current workload by CLUES, a fact that can be appreciated comparing the grey lines (the aggregated job submission pattern for both clusters) and orange (aggregated number of nodes

FiXme Note: Amanda, esta ultima frase no la entiendo. 


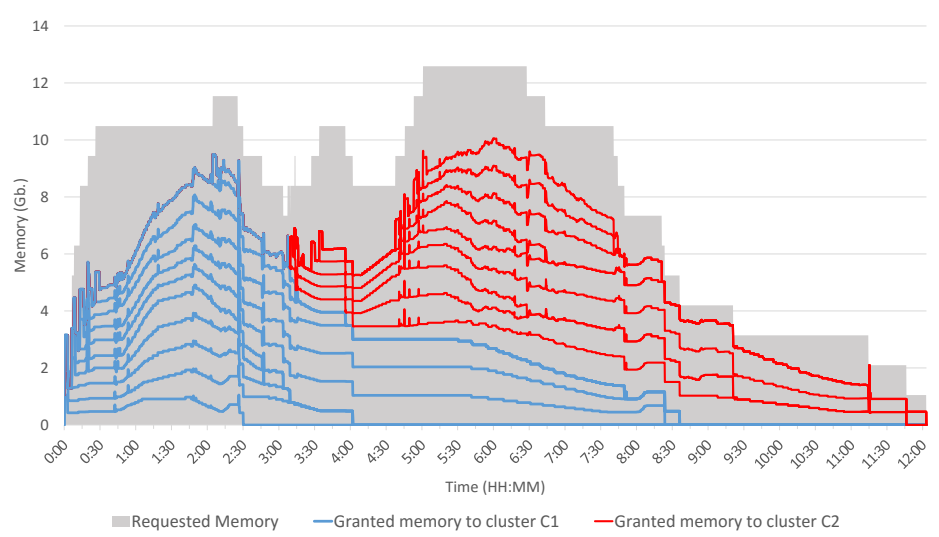

(a) Evolution of the total memory consumption.

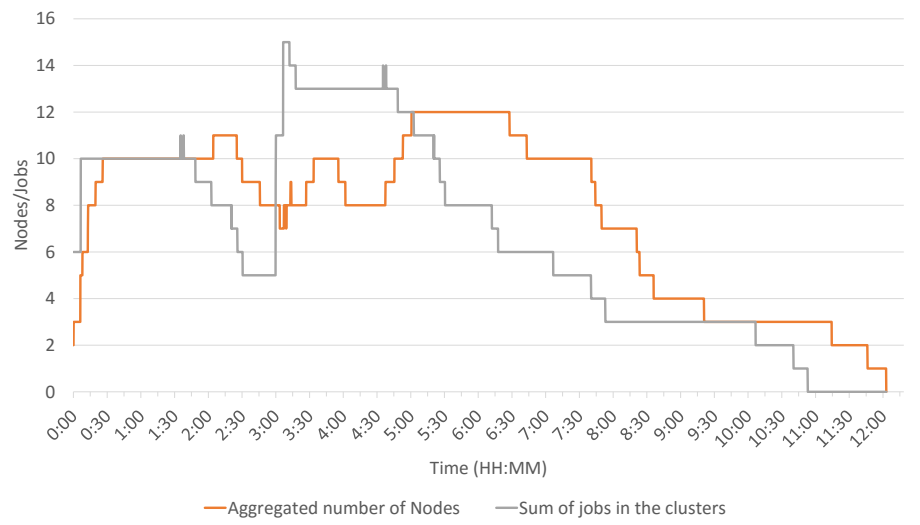

(b) Evolution of size of the clusters considering the workload pattern.

Figure 4: Performance evaluation of the proposed scenario for the complete execution.

for clusters $\mathrm{C} 1$ and $\mathrm{C} 2$ ). An appreciable delay between both lines in the graph depicts the time needed for the VMs to be deployed and configured to be integrated as a new a node of each virtual cluster, i.e., the contextualization process. This introduces a delay in the execution of jobs unless an idle node is available in the cluster, an scenario that occurred in both clusters for the last three jobs of the workload. This is the reason why the size of both clusters do not achieve the maximum number of concurrent jobs depicted in the graph close to time instante 3:00.

\section{Conclusions and further work}

This paper has introduced open-source components to manage multi-elastic datacenters, where elastic virtual clusters run on top of elastic energy-aware physical clusters. This way, the computing capabilities of the virtual clusters are dynamically changed to satisfy both the user application's computing requirements and to reduce the amount of energy consumed by the underlying physical cluster that supports an on-premises 
Cloud. For these, both horizontal elasticity, to add or remove nodes of the virtual cluster to adjust to the workload, and vertical elasticity techniques, to dynamically change the memory allocation of the VMs, have been combined. These developments can be adopted as an integrated approach to achieve better resource usage without requiring any additional effort by the users, which use the virtual computing clusters as if they were physical ones.

Future work involves addressing the dynamic allocation of CPUs for each VM, a feature that could not be initially developed due to the lack of support by the KVM hypervisor in which we based our development for CloudVAMP. OpenNebula has added support for cgroups in cooperating with KVM, thus paving the way for further research in this area. Also, the components will be evolved to support Container Orchestration Platforms instead of Cloud Management Platforms, where challenges in the area of integrated vertical and horizontal elasticity require further research activity.

\section{Acknowledgements}

The authors would like to thank the Spanish "Ministerio de Economía, Industria y Competitividad" for the project "BigCLOE' under grant reference TIN2016-79951-R.

\section{Bibliography}

\section{References}

[1] Nordugrid dataset, the grid workloads archive, Online; accessed 21-November-2016. URL: http: //gwa.ewi.tudelft.nl/datasets/gwa-t-3-nordugrid/report/.

[2] Yasuhiro Ajiro and Atsuhiro Tanaka. Improving packing algorithms for server consolidation. In Int. CMG Conference, pages 399-406. Computer Measurement Group, 2007.

[3] CERN CernVM Software appliance. Creating elastic virtual clusters. http://cernvm.cern.ch/portal/elasticclusters, 032015.

[4] Anton Beloglazov, Jemal Abawajy, and Rajkumar Buyya. Energy-aware resource allocation heuristics for efficient management of data centers for cloud computing. Future Gener. Comput. Syst., 28(5):755-768, May 2012. doi:10.1016/j.future.2011.04.017

[5] A. Bialecki, M. Cafarella, Cutting D., and OMalley O. Hadoop: A framework for running applications on large clusters built of commodity hardware. Technical report, Apache Hadoop, 2005. URL: http://hadoop.apache.org.

[6] Miguel Caballer, Marios Chatziangelou, Amanda Calatrava, Germán Moltó, and Alfonso Pérez. IM integration in the EGI VMOps Dashboard. In EGI Conference 2017 and INDIGO Summit 2017, 2017.

[7] Miguel Caballer Fernández. Gestión de infraestructuras virtuales configuradas dinámicamente. PhD thesis, Universitat Politcnica de Valncia, may 2014

[8] Amanda Calatrava, Miguel Caballer, Germán Moltó, and Alfonso Pérez. Virtual Elastic Clusters in the EGI LToS with EC3. In EGI Conference 2017 and INDIGO Summit 2017, 2017.

[9] Amanda Calatrava, Eloy Romero, Germán Moltó, Miguel Caballer, and Jose Miguel Alonso. Selfmanaged cost-efficient virtual elastic clusters on hybrid Cloud infrastructures. Future Generation Computer Systems, 61:13-25, aug 2016. URL: http://authors.elsevier.com/sd/article/ S0167739X16300024http://linkinghub.elsevier.com/retrieve/pii/S0167739X16300024, doi:10. 1016/j.future.2016.01.018

[10] Jeffrey S. Chase, David E. Irwin, Laura E. Grit, Justin D. Moore, and Sara E. Sprenkle. Dynamic virtual clusters in a grid site manager. In Proceedings of the 12th IEEE International Symposium on High Performance Distributed Computing, HPDC '03, pages 90-, Washington, DC, USA, 2003. IEEE Computer Society. URL: http://dl .acm.org/citation. cfm?id=822087.823392 
[11] T. Cioara, I. Anghel, I. Salomie, G. Copil, D. Moldovan, and A. Kipp. Energy aware dynamic resource consolidation algorithm for virtualized service centers based on reinforcement learning. In Parallel and Distributed Computing (ISPDC), 2011 10th International Symposium on, pages 163-169, 2011. doi:10.1109/ISPDC.2011.32

[12] Wesam Dawoud, Ibrahim Takouna, and Christoph Meinel. Elastic vm for cloud resources provisioning optimization. In Ajith Abraham, Jaime Lloret Mauri, JohnF. Buford, Junichi Suzuki, and SabuM. Thampi, editors, Advances in Computing and Communications, volume 190 of Communications in Computer and Information Science, pages 431-445. Springer Berlin Heidelberg, 2011. doi : 10.1007/978-3-642-22709-7_43

[13] Alfonso C. De and Blanquer I. Automatic consolidation of virtual machines in on-premises cloud platforms. In Cluster, Cloud and Grid Computing, IEEE/ACM International Symposium on, page 10701079, May 2017. doi:http://doi.org/10.1109/CCGRID.2017.128

[14] Carlos De Alfonso, Miguel Caballer, Fernando Alvarruiz, and Vicente Hernández. An energy management system for cluster infrastructures. Comput. Electr. Eng., 39(8):2579-2590, November 2013. URL: http://dx.doi.org/10.1016/j.compeleceng.2013.05.004, doi:10.1016/ j.compeleceng.2013.05.004

[15] Carlos de Alfonso, Miguel Caballer, Fernando Alvarruiz, and Germán Moltó. An Economic and Energy-Aware Analysis of the Viability of Outsourcing Cluster Computing to the Cloud. Future Generation Computer Systems (International Journal of Grid Computing and eScience), 29:704712, 2013. URL: http://www.sciencedirect.com/science/article/pii/S0167739X12001720?v=s5 doi:10.1016/j.future.2012.08.014

[16] Carlos De Alfonso, Miguel Caballer, and Vicente Hernández. Efficient Power Management in High Performance Computer Clusters. In Proceedings of the 1st International Multi-Conference on Innovative Developments in ICT, Proceedings of the International Conference on Green Computing 2010 (ICGreen 2010), pages 39-44, 2010

[17] Marcos Dias de Assuncao, Alexandre di Costanzo, and Rajkumar Buyya. Evaluating the costbenefit of using cloud computing to extend the capacity of clusters. In Proceedings of the 18th ACM International Symposium on High Performance Distributed Computing, HPDC '09, pages 141-150, New York, NY, USA, 2009. ACM. URL: http://doi.acm.org/10.1145/1551609.1551635 doi:10.1145/1551609.1551635.

[18] F. Doelitzscher, M. Held, C. Reich, and A. Sulistio. Viteraas: Virtual cluster as a service. In Cloud Computing Technology and Science (CloudCom), 2011 IEEE Third International Conference on, pages 652-657, Nov 2011. doi:10.1109/CloudCom.2011.101

[19] F. Farahnakian, P. Liljeberg, and J. Plosila. Energy-efficient virtual machines consolidation in cloud data centers using reinforcement learning. In Parallel, Distributed and Network-Based Processing (PDP), 2014 22nd Euromicro International Conference on, pages 500-507, Feb 2014. doi:10. 1109/PDP. 2014.109

[20] E. Feller, L. Rilling, and C. Morin. Energy-aware ant colony based workload placement in clouds. In Grid Computing (GRID), 2011 12th IEEE/ACM International Conference on, pages 26-33, 2011. doi:10.1109/Grid.2011.13.

[21] S.M. Ghafari, M. Fazeli, A. Patooghy, and L. Rikhtechi. Bee-mmt: A load balancing method for power consumption management in cloud computing. In Contemporary Computing (IC3), 2013 Sixth International Conference on, pages 76-80, Aug 2013. doi:10.1109/IC3.2013.6612165

[22] Marco Guazzone, Cosimo Anglano, and Massimo Canonico. Exploiting vm migration for the automated power and performance management of green cloud computing systems. In Proceedings of the First International Conference on Energy Efficient Data Centers, E2DC'12, pages 81-92, Berlin, Heidelberg, 2012. Springer-Verlag. URL: http://dx.doi.org/10.1007/978-3-642-33645-4_8, doi: 10.1007/978-3-642-33645-4_8

[23] Michael R. Hines, Abel Gordon, Marcio Silva, Dilma Da Silva, Kyung Ryu, and Muli Ben-Yehuda. Applications Know Best: Performance-Driven Memory Overcommit with Ginkgo. In 2011 IEEE Third International Conference on Cloud Computing Technology and Science, pages 130-137. IEEE, November 2011. doi:10.1109/CloudCom.2011.27

[24] Alexandru Iosup, Hui Li, Mathieu Jan, Shanny Anoep, Catalin Dumitrescu, Lex Wolters, and Dick H.J. Epema. The grid workloads archive. Future Generation Computer Systems, 24(7):672 686, 2008. URL: http://www.sciencedirect.com/science/article/pii/S0167739X08000125 doi: http://dx.doi.org/10.1016/j.future.2008.02.003

[25] Adam Litke. Manage resources on overcommitted KVM hosts. Technical report, IBM, 2011. URL: http://www.ibm.com/developerworks/library/l-overcommit-kvm-resources/

[26] P. Marshall, K. Keahey, and T. Freeman. Elastic site: Using clouds to elastically extend site 
resources. In Cluster, Cloud and Grid Computing (CCGrid), 2010 10th IEEE/ACM International Conference on, pages 43-52, May 2010. doi:10.1109/CCGRID.2010.80

[27] Moreno Marzolla, Ozalp Babaoglu, and Fabio Panzieri. Server consolidation in clouds through gossiping. In Proceedings of the 2011 IEEE International Symposium on a World of Wireless, Mobile and Multimedia Networks, WOWMOM '11, pages 1-6, Washington, DC, USA, 2011. IEEE Computer Society. doi:10.1109/WoWMoM.2011.5986483

[28] S.S. Masoumzadeh and H. Hlavacs. Integrating vm selection criteria in distributed dynamic vm consolidation using fuzzy q-learning. In Network and Service Management (CNSM), 2013 9th International Conference on, pages 332-338, Oct 2013. doi:10.1109/CNSM.2013.6727854

[29] MIT. StarCluster Elastic Load Balancer. URL: http://web.mit.edu/stardev/cluster/docs/0. 92rc2/manual/load_balancer.html

[30] Germán Moltó, Miguel Caballer, and Carlos de Alfonso. Automatic memory-based vertical elasticity and oversubscription on cloud platforms. Future Generation Computer Systems, 56:1-10, mar 2016 URL: http://dx.doi.org/10.1016/j.future.2015.10.002 doi:10.1016/j.future.2015.10.002

[31] Shuangcheng Niu, Jidong Zhai, Xiaosong Ma, Xiongchao Tang, and Wenguang Chen. Cost-effective cloud hpc resource provisioning by building semi-elastic virtual clusters. In Proceedings of the International Conference on High Performance Computing, Networking, Storage and Analysis, SC '13, pages 56:1-56:12, New York, NY, USA, 2013. ACM. URL: http://doi.acm.org/10.1145/ 2503210.2503236 doi:10.1145/2503210.2503236

[32] Cristina Bianca Pop, Ionut Anghel, Tudor Cioara, Ioan Salomie, and Iulia Vartic. A swarm-inspired data center consolidation methodology. In Proceedings of the 2nd International Conference on Web Intelligence, Mining and Semantics, WIMS '12, pages 41:1-41:7, New York, NY, USA, 2012. ACM. doi:10.1145/2254129.2254180.

[33] The GAMES research project. The games research project, January 2013. URL: http://www. green-datacenters.eu

[34] Lei Shi, J. Furlong, and Runxin Wang. Empirical evaluation of vector bin packing algorithms for energy efficient data centers. In Computers and Communications (ISCC), 2013 IEEE Symposium on, pages 000009-000015, July 2013. doi:10.1109/ISCC.2013.6754915

[35] Evangelos Tasoulas, Hå rek Haugerund, and Kyrre Begnum. Bayllocator: a proactive system to predict server utilization and dynamically allocate memory resources using Bayesian networks and ballooning. In Proceedings of the 26th international conference on Large Installation System Administration: strategies, tools, and techniques, pages 111-122. USENIX Association, December 2012.

[36] Luis Tomás and Johan Tordsson. Improving cloud infrastructure utilization through overbooking. In Proceedings of the 2013 ACM Cloud and Autonomic Computing Conference on - CAC '13, page 1, New York, New York, USA, August 2013. ACM Press. doi:10.1145/2494621.2494627

[37] GiorgioLuigi Valentini, Walter Lassonde, SameeUllah Khan, Nasro Min-Allah, SajjadA. Madani, Juan Li, Limin Zhang, Lizhe Wang, Nasir Ghani, Joanna Kolodziej, Hongxiang Li, AlbertY. Zomaya, Cheng-Zhong Xu, Pavan Balaji, Abhinav Vishnu, Fredric Pinel, JohnatanE. Pecero, Dzmitry Kliazovich, and Pascal Bouvry. An overview of energy efficiency techniques in cluster computing systems. Cluster Computing, 16(1):3-15, 2013. doi:10.1007/s10586-011-0171-x

[38] Akshat Verma, Puneet Ahuja, and Anindya Neogi. pmapper: power and migration cost aware application placement in virtualized systems. In Proceedings of the 9th ACM/IFIP/USENIX International Conference on Middleware, Middleware '08, pages 243-264, New York, NY, USA, 2008. Springer-Verlag New York, Inc.

[39] Xiaohui Wei, Haibin Wang, Hongliang Li, and Lei Zou. Dynamic deployment and management of elastic virtual clusters. In Chinagrid Conference (ChinaGrid), 2011 Sixth Annual, pages 35-41, Aug 2011. doi:10.1109/ChinaGrid.2011.31

[40] Dan Williams, Hani Jamjoom, Yew-Huey Liu, and Hakim Weatherspoon. Overdriver: handling memory overload in an oversubscribed cloud. ACM SIGPLAN Notices, 46(7):205, jul 2011. URL: http://dl.acm.org/citation. cfm?id=2007477.1952709 doi:10.1145/2007477.1952709 